\title{
Symmetry-Breaking Nanoregions in Single-Phase High Entropy Alloys Determined using Scanning Convergent Beam Electron Diffraction
}

\author{
Yu-Tsun Shao ${ }^{1}$ and Jian-Min Zuo ${ }^{1,2}$ \\ 1. Department of Materials Science and Engineering, University of Illinois, Urbana, Illinois 61801, USA \\ 2. Frederick Seitz Materials Research Laboratory, University of Illinois, Urbana, Illinois 61801, USA
}

High entropy alloys (HEAs) have attracted much research interest recently because of their unique microstructures and mechanical properties. Extensive studies showed the outstanding mechanical properties of HEAs, such as good strength and ductility, low-temperature fracture toughness, and structural stability under irradiation, which make them strong candidates for structural materials in many applications. HEAs are loosely defined as solid solution alloys which consist of five or more elements of a near-equal molar percentage [1]. This is in contrast to conventional metallic alloys that are based on one primary element and minor addition of impurities or alloying elements.

The HEAs consisted of many kinds of elements possessing different atomic sizes, which inevitably lead to distortion of the lattice. The severe lattice distortions affect the formation rule of single-phase HEAs, and impede dislocation movements which lead to pronounced solid solution strengthening [2]. The reported x-ray and neutron diffraction studies of HEAs have shown sharp Bragg peaks belonging to a simple Bravais lattice. However, the second peak of measured pair distribution function (PDF) differs from the theoretical predictions, which suggests strong lattice distortions [3].

Single crystalline $\mathrm{Al}_{0.1} \mathrm{CoCrFeNi} \mathrm{HEA}$ was studied here. To explore the local structure of HEAs, we used scanning convergent beam electron diffraction (SCBED) along with various probe sizes in a transmission electron microscope (TEM). SCBED is based on automated recording of CBED patterns on the CCD camera while scanning over the user-defined region with a nanometer-sized focused electron beam. The SCBED experiments were carried out using two different instruments, JEOL $2100 \mathrm{LaB}_{6}$ TEM and JEOL 2200FS (S)TEM with a Schottky field emission gun (FEG) and an in-column Omega energy filter. The LaB 6 instrument provides larger probes of tens of nm for SCBED over a larger field of view. The FEG instrument provides small probe sizes of $1 \mathrm{~nm}$ or less, and energy-filtering for reducing inelastic scattering effects. The symmetry quantification algorithm $[4,5]$ for the CBED whole patterns is based on the normalized crosscorrelation coefficient $(\gamma)$ of intensities between pairs of CBED discs related with symmetry operations.

We identified regions of highest symmetry are connected with regions of low symmetry from a total of 625 CBED patterns over the $25 \times 25$ scanned points. The step size of scan is comparable to the size of convergent probe. Figure 1(d) shows the most symmetrical CBED pattern of HEA along [001] zone axis using a 10nm (full-width half maximum, FWHM) probe. The orange arrows indicate the two basis mirror plane directions for symmetry quantification. Figures 1 (b) \& 1(c) show the symmetry variation map along mirror planes $(0,1) \&(1,0)$, respectively. Two representative symmetry-broken regions labeled (e) \& (f) in figure 1(b) correspond to CBED patterns. Figure 2 shows the CBED patterns and mirror maps using a $1 \mathrm{~nm}$ probe and an in-column energy-filter with energy window of $10 \mathrm{eV}$.

In summary, SCBED allows detection of the symmetry variations in a TEM sample. The detected symmetry breaking regions are inhomogeneous and localized, which is very different from the atomic stresses suggested by x-ray and neutron diffraction. The low symmetry regions are characterized by a break down in the centrosymmetry in the transmitted disk which belongs to the Laue diffraction group, which can be attributed to strain $[6,7]$. 
References:

[1] Y. Zhang et al., Prog. Mater. Sci., 61, 1 (2014).

[2] Y. Hu and J.-M. Zuo, Microsc. Microanal., 22 (Suppl 3), 1558 (2016).

[3] H. Diao et al., JOM 67, 2321 (2015).

[4] K. Kim and J.-M. Zuo, Ultramicroscopy 124, 71 (2013).

[5] Y.-T. Shao and J.-M. Zuo, Microsc. Microanal., 22 (Suppl 3), 516 (2016).

[6] B. F. Buxton et al., Philos. Trans. R. Soc. A 281, 171 (1976).

[7] This work is supported by U.S. Department of Energy under contract DEFG02-01ER45923.

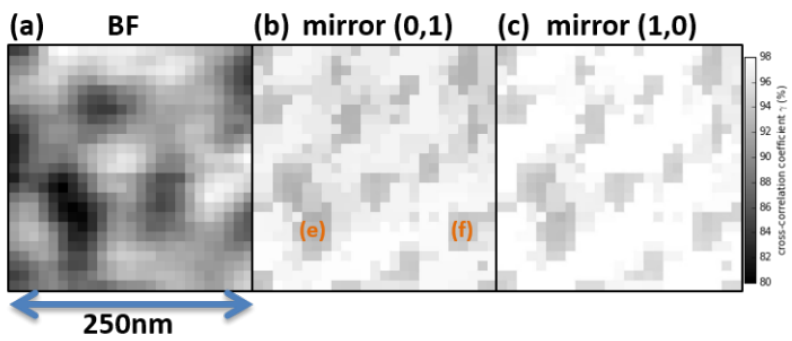

(d)

(e)

(f)

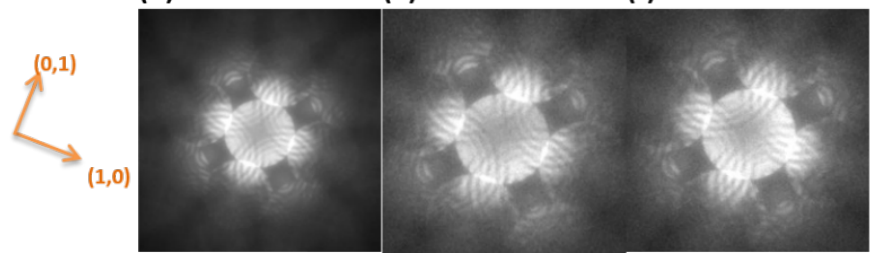

Figure 1. (a) Reconstructed bright-field image from the SCBED dataset, obtained using a 10nm probe with scanned area of $250 \mathrm{~nm} \times 250 \mathrm{~nm}$. Symmetry variation map with mirror plane along (b) $(0,1)$ and (c) $(1,0)$. (d) Representative CBED pattern which corresponds to regions with almost perfect $4 \mathrm{~mm}$ symmetry. CBED patterns with broken symmetries correspond to the labeled (e) \& (f) in (b), respectively .
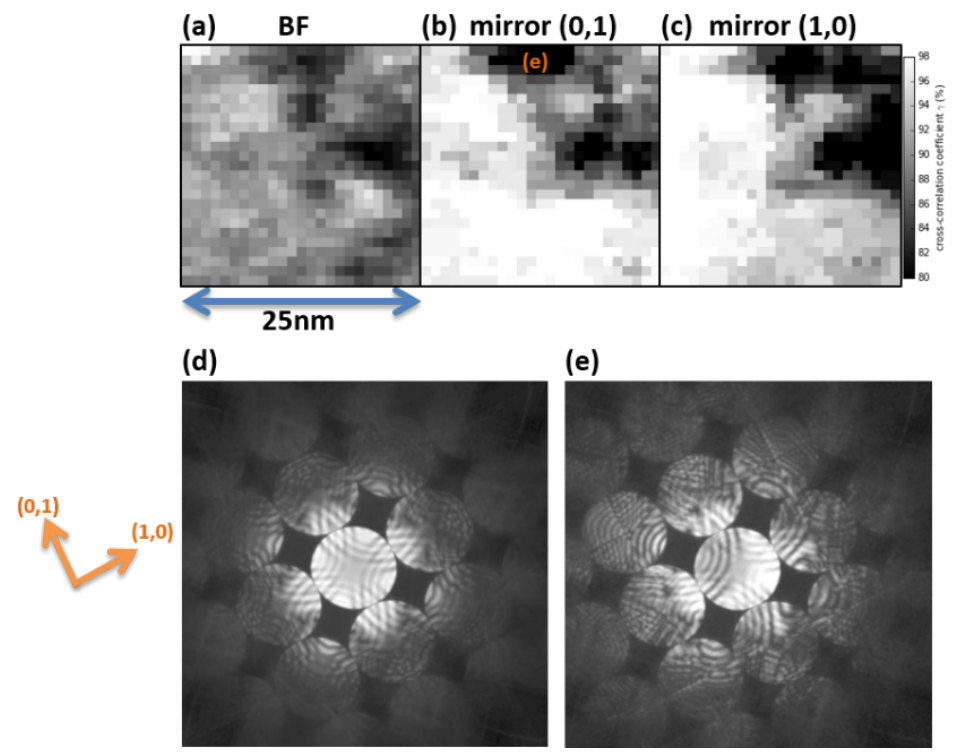

Figure 2. Figure 2. (a) Reconstructed bright-field image from the SCBED dataset with scanned area of $25 \mathrm{~nm}$ x $25 \mathrm{~nm}$, obtained using a $1 \mathrm{~nm}$ probe and in-column energy-filter with energy window of $10 \mathrm{eV}$. Symmetry variation map with mirror plane along (b) $(0,1)$ and (c) $(1,0)$. (d) Representative CBED pattern which corresponds to regions with almost perfect $4 \mathrm{~mm}$ symmetry. CBED patterns with broken symmetries correspond to the labeled (e) in (b). 